\title{
Avis de grand frais sur l'Europe
}

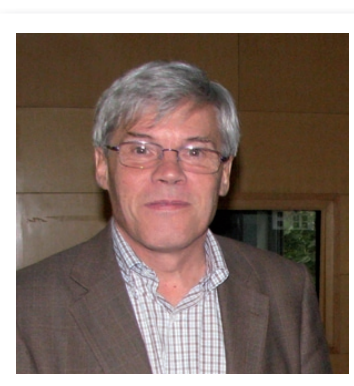

La Société Française de Physique entretient de nombreuses relations internationales, principalement en Europe et avec le Maghreb. Je voudrais m'étendre ici sur sa position à l'intérieur de la Société Européenne de Physique (European Physical Society, EPS) et sur l'évolution possible de cette dernière, suite à la récente réunion du Conseil de I'EPS à Mulhouse, fin mars.

La SFP est I'une des 18 sociétés fondatrices de I'EPS, créée en 1968 à Genève. Dès cette époque, l'EPS ignorait le " rideau de fer » et transcendait les clivages Est-Ouest, avec la participation de sociétés de physique de I'Europe de l'Est, comme celles de Roumanie, de Hongrie, de Tchécoslovaquie et même d'URSS. Elle rassemble aujourd'hui 41 sociétés de physique en Europe - au sens large, puisqu'elle inclut Israël, l'Arménie, la Géorgie et la Russie. Contrairement à la plupart des autres sociétés ou fédérations européennes (chimie, biologie...) qui ne sont que des associations de sociétés nationales, elle a présenté, dès sa création, un caractère dual : regrouper les sociétés nationales mais, en même temps, recruter des membres individuels et développer des divisions thématiques ouvertes à ces membres. Grâce à cette double structure, l'EPS a mobilisé les forces vives des sciences physiques en Europe et a permis la mise sur pied de grandes conférences européennes, de réputation internationale, dans les différentes spécialités ${ }^{(1)}$. Les divisions de la Société Européenne de Physique décernent aussi des grands prix qui, pour certains, sont des antichambres au Prix Nobel. Le développement précoce de contacts étroits entre physiciens européens d'une même discipline, à travers ces divisions et leurs bureaux, a souvent permis de devancer la politique de la Commission européenne en matière de réseaux de formation et recherche. La structure spécifique de l'EPS a aussi permis d'éviter, dans la mesure du possible, la multiplication d'associations européennes autour de thématiques ciblées, comme on en trouve dans d'autres disciplines, et de reconnaître le caractère fondamentalement interdisciplinaire des sciences physiques.

L'EPS a son siège à Mulhouse sur le campus de I'Université de Haute-Alsace. Son budget provient de contributions variées : sociétés nationales, membres individuels, membres associés. Une contribution financière très importante vient de deux sociétés nationales, I'Institute of Physics (IoP) britannique et la Société Allemande de Physique (DPG), qui comptent chacune un nombre de membres très supérieur à toutes les autres. La SFP, en dépit de son faible nombre d'adhérents, apporte une contribution non négligeable à travers sa filiale d'édition, EDP Sciences, qui publie le bulletin de I'EPS, Europhysics News.

À l'occasion du dernier Conseil de l'EPS à Mulhouse, fin mars, un comité de réflexion (d'audit ?) chargé d'évaluer les activités de la Société et de proposer des évolutions pour sa structure a été mis en place, en grande partie sous l'impulsion de I'loP et de la DPG, qui mettent en question la politique de l'EPS et l'utilisation de ses ressources financières. Un des buts exprimés par ces deux sociétés, tel qu'il apparaît dans un document diffusé au Conseil, est de faire disparaître la catégorie des membres individuels et de réduire I'EPS à un rôle d'association de type "parapluie » des sociétés nationales, avec l'objectif avoué de la confiner à une représentation de ces dernières - lobbying - à Bruxelles, auprès de la Commission européenne. Toute la richesse actuelle du fonctionnement, de type bottom-up, de l'EPS serait perdue.

Le comité de réflexion est formé de représentants des sociétés nationales, des membres individuels, des divisions et du comité exécutif de I'EPS. Il comprend en particulier la nouvelle vice-présidente, Luisa Ciffarelli, première femme élue à la tête de la Société depuis sa création. Sa position peut avoir un poids déterminant. Le comité de réflexion doit soumettre ses propositions à la fin de l'année. À suivre...

Martial Ducloy

(1) CLEO-Europe

EPS Condensed Matter Conference, EPS Plasma Physics, Europhysics Conference on High Energy Physics, etc.

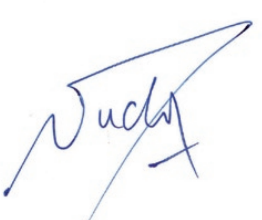

Les membres des sociétés nationales de physique, comme la SFP, peuvent devenir membres individuels de l'EPS en payant une cotisation annuelle de 20 euros. Cela leur permet de recevoir Europhysics News, d'être membre et de faire partie du Bureau d'une ou plusieurs divisions de spécialité, et d'élire les représentants des membres individuels au Conseil de I'EPS. 